\title{
BMJ Open Indigenous perspectives on concepts and determinants of flourishing in a health and well-being context: a scoping review protocol
}

\author{
Vicky Nelson (D) , ${ }^{1}$ Sarah Derrett, ${ }^{2}$ Emma Wyeth ${ }^{1}$
}

To cite: Nelson V, Derrett S, Wyeth $\mathrm{E}$. Indigenous perspectives on concepts and determinants of flourishing in a health and well-being context: a scoping review protocol. BMJ Open 2021;11:e045893. doi:10.1136/ bmjopen-2020-045893

- Prepublication history for this paper is available online. To view these files, please visit the journal online (http://dx.doi. org/10.1136/bmjopen-2020045893).

Received 14 October 2020 Revised 13 January 2021 Accepted 22 January 2021

Check for updates

(C) Author(s) (or their employer(s)) 2021. Re-use permitted under CC BY-NC. No commercial re-use. See rights and permissions. Published by BMJ.

${ }^{1} \mathrm{Ngāi}$ Tahu Māori Health Research Unit, Department of Preventive and Social Medicine, University of Otago, Dunedin, New Zealand

${ }^{2}$ Injury Prevention Research Unit, Department of Preventive and Social Medicine, University of Otago, Dunedin, New Zealand

Correspondence to Associate Professor Emma Wyeth;

emma.wyeth@otago.ac.nz

\section{ABSTRACT}

Introduction Indigenous people, including Māori in New Zealand, face many inequities in health and the determinants of health. Historically, the analysis and reporting of Indigenous health in the literature has usually taken a western medical view, often with a descriptive and deficit-oriented approach-ignoring the holistic nature of Indigenous health. This project takes a nondeficit approach and is interested in the factors that support the health and well-being of Indigenous people, including Māori. Flourishing is a recent and increasingly used term within the well-being literature; however, concepts, theories and determinants related to Indigenous flourishing are largely unknown. This scoping review aims to identify, describe and synthesise the nature and extent of the current empirical literature related to concepts, theories and determinants of Indigenous flourishing, in health and well-being contexts.

Methods and analysis Scoping review methods and guidelines included in the framework developed by Arksey and 0'Malley, and the Preferred Reporting Items for Systematic Reviews and Meta-Analyses extension for scoping reviews, will be followed for best practice and reporting of this scoping review. The literature for this review will be identified by searching the following databases: Medline (OVID), EMBASE (Ovid), Cumulative Index to Nursing and Allied Health Literature (CINAHL), Scopus, MAl journal, the Cochrane Library and Google Scholar. The research team has formulated a systematic search strategy, which will be restricted to articles published between January 1970 and May 2020 and published in the English language. Two reviewers will independently screen eligible studies for final study selection. A third reviewer will resolve any discrepancies that arise. Data from included studies will be extracted and included in thematic analysis, using a tool developed iteratively by the research team.

Ethics and dissemination Ethical approval was not required for this review. Dissemination of results will include publication in peer-reviewed journal articles, presentation of results at conferences and interactive discussions with a project expert advisory group. This scoping review also informs a larger project, examining the long-term health and flourishing of Māori, the Indigenous people of New Zealand and their whānau (families).
Strengths and limitations of this study

This scoping review will systematically identify and describe the nature and extent of the empirical literature related to Indigenous flourishing.

- This scoping review will reveal and synthesise the key concepts, theories and determinants related to Indigenous flourishing within a health context.

- The scoping review will reveal where gaps lie within the empirical literature related to Indigenous flourishing within a health context.

- A limitation of this scoping review is the restriction of included studies to those published in the English language.

- A further limitation of the scoping review is the exclusion of quality appraisal of included studies.

\section{INTRODUCTION}

Indigenous people around the world have often been found to experience inequities for a broad range of outcomes, when compared with non-Indigenous populations, including health outcomes. ${ }^{1}{ }^{2}$ Indigenous peoples experience lower life expectancies, greater morbidities and disability, greater difficulties in assessing healthcare and marked difference across many social determinants of health, compared with non-Indigenous people. ${ }^{2}$ The inequitable health status of Indigenous peoples has been called 'one of the most urgent humanitarian issues of the 21st century'. ${ }^{1}$ Māori, the Indigenous people of New Zealand, are no exception, with marked and persistent inequities in health and health outcomes, compared with non-Māori. ${ }^{3}{ }^{4}$ Māori experience lower life expectancies, and greater prevalence and incidence of many health conditions including cancer, diabetes, heart diseases, injury and disability. ${ }^{3}$ Furthermore, Māori, like other Indigenous populations, also experience poorer health outcomes and greater difficulties in accessing healthcare. ${ }^{56}$ 
To improve health and well-being outcomes for Māori, and other Indigenous peoples, policies and systems need to give attention to the health needs, cultural views and perspectives, experiences and rights of Indigenous people. ${ }^{6-8}$ In New Zealand, cutlural practices and concepts that are central to Māori perspectives of health and wellbeing are often undermined or ignored by Western views of health that dominate New Zealand policies and systems that can determine our health. ${ }^{7910}$ Western notions of disease and illness that underpin our health system are narrow and largely individualised, assuming health 'symptoms' are treated with individual 'cures'. ${ }^{11}$ While there has been a move towards the acknowledgement and understanding of the social determinants of health and their impact, which has shifted the 'blame' of illness and disease from the individual to political and economic systems that uphold persistent inequities in health and disease. ${ }^{11-13}$ However, the focus is often still deficit based, for Māori and many other Indigenous communities around the world, ${ }^{11}{ }^{14}$ and does not often include an Indigenous worldview.

For Indigenous communities, holistic models of health are not only concerned with physical health and wellbeing but also refer to social, emotional, spiritual and cultural dimensions. ${ }^{15}$ Indigenous views of health are not focused solely on individuals, but also on the collective. For Māori, it includes the health and well-being of whānau (family), hapū (sub-tribe) and iwi (tribe). To truly improve Māori and Indigenous health, Indigenous views of health and well-being must be actively recognised and included in health system and policy planning and development. While there are already existing concepts that have been employed to describe and measure health and health outcomes such as 'well-being'; 'quality of life'; 'coping mechanisms' and 'resilience', ${ }^{16}$ flourishing may be more relevant for describing long-term health and well-being outcomes, particularly for Māori and other Indigenous peoples. This is because flourishing can include multidimensional factors of health, is nondeficit oriented, may avoid blaming individuals, and can incorporate broader social and structural determinants of health. ${ }^{15-17}$ Additionally, flourishing may recognise the struggles that marginalised people, particularly Indigenous people, face. ${ }^{1517}$

In a health context, definitions of flourishing largely exist within the Western mental and psychological health fields. Here, flourishing is defined as when one is 'illed with positive emotion and to be functioning well psychologically and socially'. ${ }^{18} 19$ However, flourishing in a health and well-being context must go beyond the individual-level absence of disease or illness, to account for the resources required for individuals and collectives, to face adversity such as illness and disease and ultimately flourish in life. ${ }^{1617}$ Flourishing can look over a lifecourse, rather than a single point in time or a final goal. ${ }^{16} 18$

Flourishing is increasingly being used in the literature, but its definition is inconsistent, context dependent, and often non-Indigenous in perspective. ${ }^{17} \mathrm{An}$ in-depth understanding of flourishing and the indicators and determinants of flourishing from an Indigenous perspective is important. Such an understanding has the potential to drive important policy and funding decisions, that affect Indigenous health and well-being (and life). For some time now, researchers and organisations have been collecting and reporting deficit-based well-being indicators, which describe the inequities in Indigenous health as a "product of causes and effects'. ${ }^{11}$ This has not considered a holistic and Indigenous view of health or the factors that account for Māori and indigenous peoples to promote their well-being and to flourish. ${ }^{11} 1517$ This has led to ineffective policies and practices that have not improved health outcomes for Māori or Indigenous peoples, and at times have only aided the inequities to exist. ${ }^{11}$ To understand flourishing from an Indigenous perspective and worldview, we must examine the current Indigenous knowledge base.

A scoping review methodology is being employed to examine the current empirical literature regarding models, frameworks, theories and the determinants of Indigenous flourishing in a health and well-being context, from an Indigenous perspective. A scoping review of the literature allows for the synthesis of the literature, to explore, map and understand the extent of the currently available literature. ${ }^{20-22}$ Furthermore, scoping reviews allow for the identification and summary of knowledge gaps, to assist with making recommendations for future research. $^{20-22}$

\section{OBJECTIVES}

The overall aim of this scoping review is to identify, describe and synthesise the key frameworks, models, theories and determinants of Indigenous flourishing in health and well-being contexts.

\section{METHODS/DESIGN}

Scoping reviews are useful when wanting to examine a broadly covered research area, to systematically map the literature and to identify key concepts, theories, evidence and research gaps. ${ }^{20}{ }^{21}$ When designing the protocol for this scoping review, we adhered to the scoping review methods developed by Arksey and O'Malley ${ }^{20}$ and the subsequent refinement of these methods outlined by others. ${ }^{21} 22$ The Arksey and O'Malley ${ }^{20}$ framework outlines a 5-stage scoping review methodology, which includes (1) identifying the research question, (2) identifying the relevant studies, (3) study selection, (4) charting the data and (5) collating, summarising and reporting the results. These stages are discussed below in relation to our scoping review. The Preferred Reporting Items for Systematic Reviews and Meta-Analyses (PRISMA) PRISMA extension for scoping reviews (PRISMA-ScR) ${ }^{23}$ checklist will be followed. In addition, the project and scoping review will be guided by kaupapa Māori research principles (Indigenous research principles), ${ }^{24} 25$ and we 
will employ the Consolidated criteria for strengthening the reporting of health research involving Indigenous Peoples (CONSIDER) framework for the reporting of Indigenous health research. ${ }^{26}$

\section{Stage 1: identifying the research question}

In following the scoping review protocol outlined by Arksey and $\mathrm{O}^{\prime}$ Malley, ${ }^{20}$ we took an iterative approach when developing the guiding research questions for this scoping review. This iterative process aided in developing guiding research questions that considered the concepts (flourishing), context (health and well-being) and target population (Indigenous populations) of interest in this scoping review. The process of developing these guiding research questions aided in refining the focus of the review as well as establishing a strategy to identify relevant studies. This scoping review sets out to answer the following questions:

1. What are the frameworks, models and/or theories related to Indigenous flourishing in a health and wellbeing context?

2. What are the determinants of Indigenous flourishing in a health and well-being context?

\section{Stage 2: identifying the relevant studies}

The scoping review methodology is useful in addressing broad research questions, however, it still requires parameters in order to achieve a comprehensive and rigorous review of the existing literature. The parameters included: the search strategy, eligibility criteria of studies, and the databases to be searched. These parameters were developed by the research team, in conjunction with an experienced University of Otago Subject Librarian.

\section{Search strategy}

To comprehensively search and identify empirical literature, a limited search of relevant databases has been performed by $\mathrm{VN}$, with a review of all text words in title and abstracts and of index and keyword terms to describe key articles by the research team. These were then used to develop a list of keywords, which were then used to develop a search strategy in Medline (Ovid) (see table 1). This was then adapted for other databases.

A secondary search will then be undertaken using all identified primary and secondary search terms, keywords and subject headings in the databases listed below. The search will be restricted using the filtering methods using the eligibility criteria. Boolean search terms ('and' and 'or') will be used during searches, to refine and combine terms, concepts and keywords. The reference lists of all included articles will also be searched for additional studies. On completion, the searches from all databases, search engines and referrals will be documented, and references will be imported into reference manager Endnote X8.2. ${ }^{27}$

\section{Databases}

Electronic databases that will be used to identify relevant studies include Medline (OVID), EMBASE (Ovid), the
Table 1 Ovid (Medline) search strategy

Search terms entered into

\begin{tabular}{llr} 
Line number & Ovid(Medline) & \multicolumn{1}{c}{ Results } \\
\hline 1 & Maori.af. & 3470 \\
2 & M?ori.af. & 58607 \\
\hline & $\begin{array}{l}\text { First nations/ or Indigenous/ or } \\
\text { native/ or aborigin*.af. }\end{array}$ & 253920 \\
4 & $\begin{array}{l}\text { Flourishing/ or human } \\
\text { flourishing.af. }\end{array}$ & 1478 \\
5 & Mauri ora/ or Wairua & 21 \\
6 & $\begin{array}{l}\text { Sustained health/ or prosperity/ } \\
\text { or aspiration/ or develop*/ or } \\
\text { resilience/ or well-being/ or }\end{array}$ & \\
& well-being.af.
\end{tabular}

\begin{tabular}{|c|c|c|}
\hline 7 & 1 or 2 & 58607 \\
\hline 8 & 4 or 5 & 1499 \\
\hline 9 & 7 AND 8 & 12 \\
\hline 10 & 6 or 8 & 5114629 \\
\hline 11 & 7 and 10 & 12496 \\
\hline 12 & 3 or 7 & 31170 \\
\hline 13 & 10 and 12 & 67307 \\
\hline 14 & $\begin{array}{l}\text { Concepts/ or part/ or } \\
\text { perception/ or characteris*/ } \\
\text { or defin*/ or meaning/ or } \\
\text { describe/ or cause/ or element/ } \\
\text { or factor/ or foundation/ or } \\
\text { contribut*/ or variabl*.af. }\end{array}$ & 8423135 \\
\hline 15 & Determine/ or determinants.af. & 1545752 \\
\hline 16 & $\begin{array}{l}\text { Theories/ or theory/ or } \\
\text { framework/ or model.af. }\end{array}$ & 2357378 \\
\hline 17 & Health/ or hauora.af. & 4750877 \\
\hline 18 & 14 or 15 or 16 & 10482715 \\
\hline 19 & 1 or 2 or 3 & 311170 \\
\hline 20 & 4 or 5 & 1499 \\
\hline 21 & 4 or 5 or 6 & 5114629 \\
\hline 22 & 4 and 7 and 18 & 21 \\
\hline 23 & 18 and 19 and 20 & 0 \\
\hline 24 & 4 and 7 and 18 & 13 \\
\hline 25 & 4 and 18 and 19 & 23 \\
\hline 26 & 17 and 19 and 20 & 15532 \\
\hline 27 & $\begin{array}{l}\text { Limit } 26 \text { to (humans and } \\
\text { English) }\end{array}$ & 6811 \\
\hline 28 & $\begin{array}{l}\text { Limit } 22 \text { to (humans and } \\
\text { English) }\end{array}$ & 12 \\
\hline 29 & $\begin{array}{l}\text { Limit } 24 \text { to (humans and } \\
\text { English) }\end{array}$ & 7 \\
\hline 30 & $\begin{array}{l}\text { Limit } 25 \text { to (humans and } \\
\text { English) }\end{array}$ & 20 \\
\hline 31 & Limit 9 to (humans and English) & 13 \\
\hline
\end{tabular}


Table 2 Inclusion and exclusion criteria for title and abstract screening, developed a priori

\begin{tabular}{|c|c|}
\hline Inclusion criteria & Exclusion criteria \\
\hline $\begin{array}{l}\text { Flourishing or flourishing- } \\
\text { related secondary keywords or } \\
\text { terms, are the main concept } \\
\text { Includes an Indigneous } \\
\text { population } \\
\text { And at least one other: } \\
\text { - Health and well-being } \\
\text { - Concept, theory, framework } \\
\quad \text { or model } \\
\text { - Determinant(s) } \\
\text { Published in the English } \\
\text { language } \\
\text { Human participants } \\
\text { All ages } \\
\text { Published between } 1970 \text { and } \\
2020 \\
\text { Empirical publications; } \\
\text { including qualitative and } \\
\text { quantitative studies. }\end{array}$ & $\begin{array}{l}\text { Not related to: } \\
\text { Flourishing } \\
\text { Health and well-being } \\
\text { Indigenous population } \\
\text { Human } \\
\text { Published outside of } \\
1970-2020 \\
\text { Not published in the } \\
\text { English language } \\
\text { Protocol publications } \\
\text { - Grey literature sources }\end{array}$ \\
\hline
\end{tabular}

Cumulative Index to Nursing and Allied Health Literature (CINAHL), Scopus, MAI journal, the Cochrane Library as well as the academic search engine, Google Scholar. The reference lists of included studies will be searched, as well as citations for key studies, for additional studies.

\section{Eligibility criteria}

Inclusion and exclusion criteria were developed, again using an iterative process, which included discussions within the research team and between the team and librarian and growing familiarity with the literature. The inclusion and exclusion criteria will be used to guide the searching of databases and when reviewing articles include the following and are outlined in table 2 .

The inclusion criteria include:

- Flourishing or flourishing-related terms be the main concept.

- Includes or references an Indigenous population.

- Human participants.

- All ages.

- Published between 1970 and 2020.

- Published in the English language.

The exclusion criteria include:

- Not related to flourishing.

- Not related to health or well-being.

- Not include an Indigenous population.

- Be a protocol publication or grey literature source.

\section{Stage 3: study selection}

As previously mentioned, all studies retrieved from the study searches will be exported to Endnote X8.2. Duplicate records will then be removed, using both the Endnote 'de-duping' function and manual scanning by $\mathrm{VN}$. The remaining records will be included for title and abstract screening.
Title and abstract screening will occur in a two-step approach. The first step will involve two researchers independently reviewing all titles ( $\mathrm{VN}$ and $\mathrm{EW})$, with eligibility for the second stage of abstract screening based on the defined inclusion and exclusion criteria outlined above. Any changes to these criteria will be discussed with the wider research team. If there is any uncertainty, that study will be included for abstract screening in the second stage.

The second stage of the study selection process involves title and abstract review, by two researchers. Two researchers (VN and EW) will independently pilot the selection process and eligibility criteria, with adaptions as required, on approximately the first 10 studies (see table 2). When consensus has been reached, two researchers (VN and an experienced research associate) will independently review the abstracts of the remainder of the studies, applying the refined eligibility criteria for studies to be retained for full-text review. When it is unclear from the title and abstract review, if the inclusion and exclusion criteria outlined in table 2 are met, the paper will also proceed to full-text review. Throughout the selection process, the two researchers will meet regularly to assess the agreement on the review process and included and excluded studies for full-text review. A third researcher (SD and/or EW) will be consulted when there is disagreement in the inclusion or exclusion of studies for full-text review until a consensus has been reached.

The eligibility criteria for the inclusion and exclusion of studies for full-text data extraction has been developed a priori by the research team. Studies will be retained for full-text data extraction if they meet the following criteria outlined in table 2, including (1) concepts, theories, frameworks or determinants of flourishing (or related keywords) as a main concept, (2) an Indigenous population, (3) a health or well-being context, (4) a fulltext empirical literature source, (5) published between 1970 and 2020, (6) exclude protocols and grey literature sources (eg, conference abstracts and proceedings; government documents, theses and dissertation papers; editorial and opinion pieces).

\section{Stage 4: charting the data}

The data extraction and charting stage will follow the methods outlined by Arksey and $\mathrm{O}^{\prime} \mathrm{Malley}^{20}$ and the advancements of those methods outlined by subsequent authors. ${ }^{21} 22$ Furthermore, charting of the data will adhere to the recommendations set out in the CONSIDER statement by Huria et al, ${ }^{26}$ which outlines criteria for the reporting of health research that involves Indigenous populations. A standardised data extraction framework will be developed by the authors, in an iterative process and with growing familiarity with the literature. The framework will include standard information (such as author, year of publication, type of publication, study objectives) and additional information to examine the concepts and determinants of flourishing from an Indigenous perspective, for descriptive analysis. All information 
will be collated and exported to an excel spreadsheet. In addition, qualitative data management software $\mathrm{NVivo}^{28}$ will be used to thematically analyse ${ }^{29} 30$ all studies to examine and synthesise the concepts and determinants of flourishing.

Data extraction will first be piloted on the first 10 studies by two researchers (VN and $\mathrm{EW}$ ). This will be consulted and discussed with a third researcher (SD). Modifications to the data extraction framework may be made, to ensure that the data extraction is consistent with the aims and research questions of the scoping review. Once consensus has been reached, a single researcher (VN) will perform the remainder of the data extraction, with regular consultation with other researchers (EW and SD). Once extraction has been completed, a second researcher (EW) will review the data, and any disagreement will be discussed with a third researcher (SD).

\section{Stage 5: collating, summarising and reporting the results}

Stage 5 of the scoping review will represent the most extensive stage of the review process. The purpose of this scoping review is to aggregate and synthesise the key frameworks, models, theories and determinants of Indigenous flourishing, in a health and well-being context, within the current literature. This will be achieved in a two-stage approach. The first stage will involve aggregation and descriptive analysis of the data imported into an Excel spreadsheet, according to the CONSIDER statement. ${ }^{26}$ The second step will involve thematic analysis of included studies to examine the current evidence base regarding Indigenous flourishing, within a health and well-being context. This will then be used to develop a framework or model of the key concepts, theories and determinants from the current evidence base and highlight any gaps in the empirical literature.

Steps 1 and 2 of stage 5 will take an iterative and collaborative approach. A single researcher (VN) will carry out steps 1 and 2, with regular consultation, review and consideration throughout after stage 5 , by the other researchers (EW and SD). Included within stage 5, and particularly when summarising and reporting the results, it is important to consider the broader context of the scoping review results and the overall implication of these findings. The PRISMA reporting guidelines for scoping reviews (PRISMA-ScR) and the CONSIDER ${ }^{26}$ statement for reporting Indigenous health research will be used to guide collating and reporting of results.

\section{Stage 6: consultation}

The consultation stage of the scoping review outlined by Arksey and O'Malley ${ }^{20}$ provides an important opportunity to gain involvement and insight into stakeholders and key informants that go beyond the literature. In accordance with kaupapa Māori research principles and the CONSIDER statement, consultation with, and involvement of Indigenous stakeholders, is a necessary and crucial element to all research that involves Māori and other Indigenous populations. This is to ensure that research and research findings are relevant to Māori and other Indigenous populations.

This scoping review is a part of larger project, which includes an expert advisory group consisting of Māori health and research experts, who will be consulted concerning the scoping review findings and potential implications. Further, the scoping review findings will help inform qualitative interviews as part of the larger study. Interview participants will also be consulted on the key findings and potential implications of the scoping review.

\section{Patient and public involvement \\ No patient involvement}

\section{ETHICS AND DISSEMINATION}

Ethical approval was not required for this scoping review. To the best of our knowledge, this scoping review is the first to systematically identify and describe the extent and nature of the current literature concerning the frameworks, models, theories and determinants of Indigenous flourishing, in a health and well-being context. Therefore, this scoping review will be of interest to researchers (eg, health, Māori, Indigenous), clinicians, key New Zealand organisations (eg, Accident Compensation Corporation, the Ministry of Health, the Ministry of Social Development), Māori health organisations, as well as internationally, given the paucity of literature related to Indigenous flourishing. Our dissemination strategy will include the publication of this review in an open-access peer-reviewed journal, presentations at conferences, to the projects advisory groups and those working within the Māori and Indigenous health sector. This scoping review also represents a first step to inform a larger project, which will examine the determinants of long-term flourishing of injured Māori and their whānau (family).

\section{Twitter Emma Wyeth @ehwyeth}

Acknowledgements We would like to acknowledge Christy Ballard, the University of Otago, Department of Preventive and Social Medicine subject librarian for her assistance in the development of the search strategy and related terms.

Contributors VN completed an initial draft of the scoping review protocol and led the development of the research questions and methods. EW and SD jointly helped conceptualise the scoping review and guided the development of the research questions and methods and provided feedback on multiple iterations of this manuscript.

Funding This work was supported by Health Research Council (HRC) of New Zealand, grant number [19/325].

Competing interests None declared.

Patient and public involvement Patients and/or the public were not involved in the design, or conduct, or reporting, or dissemination plans of this research.

Patient consent for publication Not required.

Provenance and peer review Not commissioned; externally peer reviewed.

Open access This is an open access article distributed in accordance with the Creative Commons Attribution Non Commercial (CC BY-NC 4.0) license, which permits others to distribute, remix, adapt, build upon this work non-commercially, and license their derivative works on different terms, provided the original work is properly cited, appropriate credit is given, any changes made indicated, and the use is non-commercial. See: http://creativecommons.org/licenses/by-nc/4.0/. 
ORCID iD

Vicky Nelson http://orcid.org/0000-0002-2765-9601

\section{REFERENCES}

1 Stephens C, Porter J, Nettleton C, et al. Disappearing, displaced, and undervalued: a call to action for Indigenous health worldwide. Lancet 2006;367:2019-28.

2 Stephens C, Nettleton C, Porter J, et al. Indigenous peoples' health-why are they behind everyone, everywhere? Lancet 2005;366:10-13.

3 Ajwani Set al. Decades of disparity: ethnic mortality trends in New Zealand 1980-1999. Wellington: Ministry of Health, 2003.

4 Reid P, Robson B, Inequities UH. Hauora: Maori Standards of Health IV. In: Robson B, Harris R, eds. A study of the years 20002005. Wellington, NZ: Te Rōpū Rangahau Hauora a Eru Pōmare, 2007: 3-10.

5 Wyeth EH, Derrett S, Harwood M. Māori disability outcomes: pathways and experiences after injury. Health Research Council of New Zealand, 2014

6 Harwood M. Rehabilitation and Indigenous peoples: the Māori experience. Disabil Rehabil 2010;32:972-7.

7 Ellison-Loschmann L, Pearce N. Improving access to health care among New Zealand's Maori population. Am J Public Health 2006;96:612-7.

8 Wyeth EH, Derrett S, Hokowhitu B, et al. Rangatiratanga and Oritetanga: responses to the Treaty of Waitangi in a new Zealand study. Ethn Health 2010;15:303-16.

9 Cram F, Smith L, Johnstone W. Mapping the themes of Maori talk about health. New Zealand Medical Journal 2003;116:1.

10 Tribunal W. Hauora report on stage one of the health services and outcomes inquiry. Wellington, New Zealand: Waitangi Tribunal, 2019.

11 Rountree J, Smith A, Addie Smith MSW J. Strength-based well-being indicators for Indigenous children and families: a literature review of Indigenous communities' identified well-being indicators. Am Indian Alsk Native Ment Health Res 2016;23:206-20.

12 Robson B, Cormack D, Cram F. Social and economic indicators. In: Hauora: Maori standards of health IV. A study of the years, 2000, 2005: 21-32.

13 Chin MH, King PT, Jones RG, et al. Lessons for achieving health equity comparing Aotearoa/New Zealand and the United States. Health Policy 2018;122:837-53.
14 George E, Mackean T, Baum F, et al. Social determinants of Indigenous health and Indigenous rights in policy: a scoping review and analysis of problem representation. Int Indig Policy $J$ 2019;10:1-25.

15 Durie M. Ngā Tini Whetū: navigating Māori futures. Huia Publishers, 2011.

16 Edgar A, Pattison S. Flourishing in health care. Health Care Anal 2016;24:161-73.

17 Kingi TKet al. Te Puawaitanga o Ngā Whānau: 6 Markers of Whānau Flourishing. Palmerston North, New Zealand: Massey University, 2014.

18 Keyes CLM. The mental health continuum: from languishing to flourishing in life. J Health Soc Behav 2002;43:207-22.

19 Keyes CLM. Mental illness and/or mental health? investigating axioms of the complete state model of health. J Consult Clin Psychol 2005;73:539-48.

20 Arksey H, O'Malley L. Scoping studies: towards a methodological framework. Int J Soc Res Methodol 2005;8:19-32.

21 Levac D, Colquhoun H, O'Brien KK. Scoping studies: advancing the methodology. Implement Sci 2010;5:69.

22 Colquhoun HL, Levac D, O'Brien KK, et al. Scoping reviews: time for clarity in definition, methods, and reporting. J Clin Epidemiol 2014;67:1291-4.

23 Tricco AC, Lillie E, Zarin W, et al. PRISMA extension for scoping reviews (PRISMA-ScR): checklist and explanation. Ann Intern Med 2018;169:467-73.

24 Cram FTolich M, ed. Rangahau Māori: Tona tika, tona pono - The Validity and Integrity of Mãori Research, in Research Ethics in Aotearoa New Zealand. Auckland, New Zealand: Pearson Education New Zealand Limited, 2001: 35-52.

25 Walker S, Eketone A, Gibbs A. An exploration of kaupapa Māori research, its principles, processes and applications. Int J Soc Res Methodol 2006;9:331-44.

26 Huria T, Palmer SC, Pitama S, et al. Consolidated criteria for strengthening reporting of health research involving Indigenous peoples: the CONSIDER statement. BMC Med Res Methodol 2019;19:173.

27 The Endnote Team. Endnote. Philadelphia, PA: Clarivate, 2018.

28 Bergin M. NVivo 8 and consistency in data analysis: reflecting on the use of a qualitative data analysis program. Nurse Res 2011;18:6-12.

29 Clarke V, Braun V, Hayfield N. Thematic analysis. In: Qualitative psychology: a practical guide to research methods, 2015: 222-48.

30 Wolcott HF. Transforming qualitative data: description, analysis, and interpretation. Sage, 1994 